\title{
CHILDHOOD IMMUNIZATION; AWARENESS AMONG PREGNANT WOMEN IN PAKISTAN
}

1. Intern at Aga Khan University Hospital.

Karachi

2. Associate Professor MBBS, FCPS in Physiology Bahria University of Medical and Dental College.

Karachi

3. House Officer at Jinnah Medical College Hospital,

Karachi

4. Intern at Aga Khan University

Hospital

Karachi

Correspondence Address:

Dr. Iram Saddiqa Aamir

(FCPS in Physiology)

Associate professor

Physiology department

Bahria University Medical and Dental

College

Karachi

Article received on:

21/10/2015

Accepted for publication:

03/12/2015

Received after proof reading:

$13 / 01 / 2016$

\section{Dr. Syed Aoun Muhammad Jafri', Dr. Iram Saddiqa Aamir ${ }^{2}$, Dr. Marium Abid ${ }^{3}$, Dr. Maliha Hanif ${ }^{4}$}

\begin{abstract}
Introduction: Childhood immunization remains one of the most important ways of preventing outbreaks of deadly diseases. Even with full-fledged active Expanded programme of immunization (EPI) initiated in Pakistan, the percentage of children getting vaccinated remain far below our required levels. Infant mortality in Pakistan is highest among SAARC countries, which at present stands at 70 deaths per 1,000 live births, according to a report compiled by the Society for the Protection of the Rights of the Child (SPARC). Objective: This study was carried out to assess mother's education, knowledge and level of awareness of childhood immunization among the pregnant women. Study design: Cross sectional descriptive study. Setting: Jinnah Medical and Dental College Karachi in department of community Health sciences (CHS). Study period: April 2012 to June 2012. Methods: A total number of Four hundred pregnant (400) women were interviewed in both OPDs and wards of Lady Dufferin Hospital and JMCH Karachi. The questionnaire contained a total of nineteen questions. All of them were self-asked in local language. Results: A significant number of child bearing women (81.5\%) had good knowledge and aptitude about childhood immunization and are likely to get their children vaccinated. Conclusion: The higher the people are aware regarding childhood immunization, the more they are likely to get their children vaccinated. The level of awareness invariably correlated with the level of education of pregnant women. This tells us the literacy rate along with efficient immunization campaigns can help us fight against the war of high infant mortality rate against curable diseases.
\end{abstract}

Key words: $\quad$ Awareness, childhood immunization, pregnant women.

Article Citation: Jafri SAM, Aamir IS, Abid M, Hanif M. Childhood immunization; awareness among pregnant women in Pakistan. Professional Med J 2016;23(1):071075. DOI: $10.17957 / T P M J / 16.3137$

\section{INTRODUCTION}

Immunization has been regarded as the most costeffective intervention for child health promotion by the World Health Organization (WHO) and reduces both the morbidity and mortality associated with infectious diseases, thus providing a healthy childhood; and reducing poverty and suffering. ${ }^{1}$ Vaccination or immunization uses the body's usual defence mechanism - the immune response - building resistance to specific infections. ${ }^{1}$ Nine diseases can be prevented by routine childhood immunization - diphtheria, tetanus, whooping cough, poliomyelitis (polio), measles, mumps, rubella, Haemophilus influenzae type b (Hib) and hepatitis B..$^{1,2}$ All of these diseases can cause serious complications and sometimes death. ${ }^{2}$

According to statistics by WHO in 2008 , around 1.5 millions death among children $<5$ years of age were due to contagious diseases which can be prevented by vaccination. ${ }^{3}$ Despite this, various preventable diseases remain the most common cause of childhood mortality with an estimated three million deaths each year. ${ }^{3}$ Uptake of vaccination services is dependent not only on provision of these services but also on other factors including knowledge and education of mothers being the critical one..$^{1,2}$

Childhood Immunization starts right from birth and all previous relevant studies have been done on mothers already having children. ${ }^{4,5} \mathrm{~A}$ number of immunizations are required in the first few years of a child's life to protect the child against the most serious infections of childhood. ${ }^{2}$ In the first months of life, a baby is protected from most infections by antibodies from her or his mother which are transferred to the baby during pregnancy. ${ }^{6}$ When 
these antibodies wear off, the baby is at risk of serious infections and so the first immunizations are given before these antibodies have worn off with time leaving the baby unprotected. ${ }^{7}$

A mother is considered to be the most responsible person in a child's life. ${ }^{8}$ Knowledge and known importance about childhood immunization in mothers not only increases the chances of the child to get immunized but also increases chances of the child to be prevented from developing grave lethal diseases. ${ }^{1,8}$

We postulated that an expecting mother may be more sensitized to the well being of her coming baby and therefore, if adequately aware, is more likely to commence immunization of her child. In light of this background, the purpose of this crosssectional survey study was to develop an interview based questionnaire and assess the awareness of childhood immunization in pregnant women.

\section{METHODS}

We conducted a qualitative, cross-sectional descriptive study at OPD and wards of Lady Dufferin Hospital and Jinnah Medical College Hospital, Korangi Karachi from April 2012 to June 2012. Duration of the study was three months and the sampling technique was purposive. Permission and consent for conducting the survey was taken from the respective hospitals and the attending patients.

A total number of 400 pregnant women were surveyed via questionnaires that consisted of 19 questions. The questionnaire was conducted in local language Urdu for ease of understanding of the masses included in the study. The questions were asked individually by a group of 6 members supervised by the Head of Department of Community Medicine, Jinnah Medical and Dental College Karachi.

Questions were asked in order to have a fair idea about their knowledge, education and aptitude regarding significance of immunization. It was also made ascertained how did they get this information regarding immunization and how effectively they would imply on it. The questionnaire was designed so that it also determined the trimester of their pregnancy, education level of pregnant women and the attitude of their husbands which was correlated with the chances of these pregnant women for getting their children vaccinated. Women who already had children were also asked about the immunization status of their previous children.

\section{STATISTICAL ANALYSIS}

The data was collected and analyzed in SPSS version 16. Age was presented as Mean+ SD. Frequencies and percentages were computed using all categorical variables like literacy rate and level of knowledge.

\section{RESULTS}

In our study, total no. of 400 pregnant women were enrolled. Their ages were $28+10$. Our results showed that a total number of 326 pregnant women out of 400 (81.5\%) were self aware about the programs and importance of childhood immunization. 31 women (15.2\%) had no knowledge about childhood immunization and 13(3.2\%) were not sure about it.

Fig 1 shows that the most frequent source of information regarding immunization programs remained the Doctors of pregnant women. 161 women $(40.2 \%)$ mentioned that doctors were their first source of information about childhood vaccination and they were the one who educated and convinced them for getting their children vaccinated against dreadful diseases. Family members were the second most important source of information and a total of 112 women (28.0\%) reported them as their first source of information.

Vaccination regarding separate disease entities included in EPI schedule of Pakistan was also questioned about. This was to determine the extent of their knowledge regarding the EPI program. From table I, it is clear that Polio was the most known vaccination and 329 pregnant women $(82.2 \%)$ confirmed that vaccine against this disease is included in EPI program. This could probably due to massive polio campaigns that 


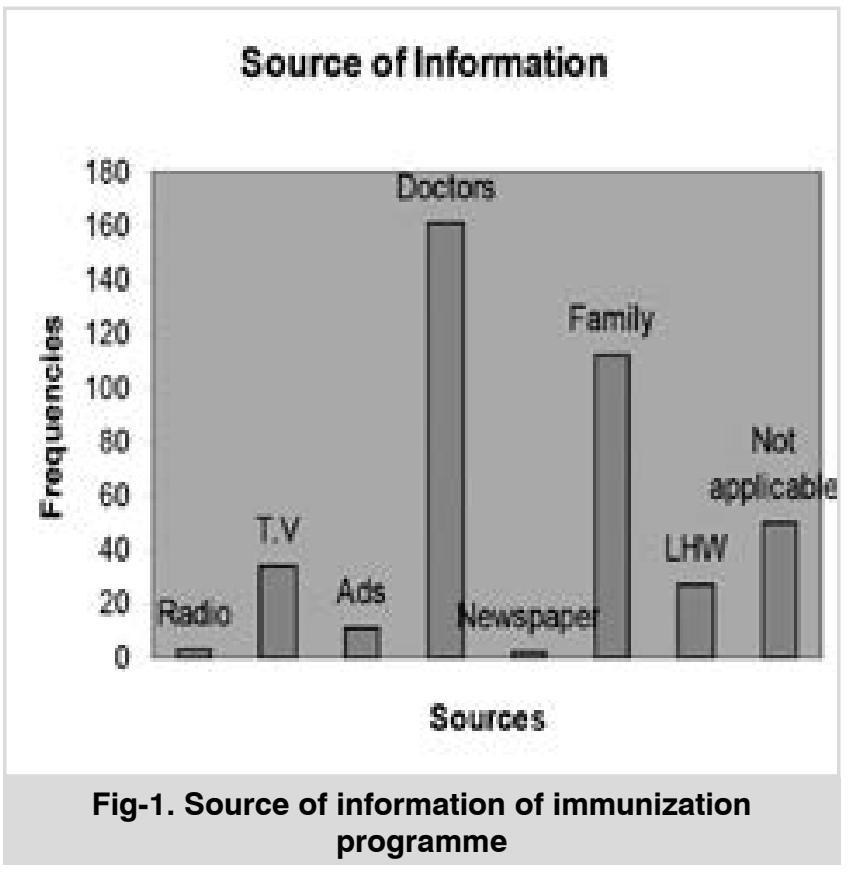

are taking place all across Pakistan and helping in the awareness of importance of prevention of this disease. Only 71 women (17.8\%) were not aware about vaccine against polio. Measles and Hepatitis were also reported as vaccines included in EPI (59.5\%) and (51.0\%) respectively.

There were certain misconceptions in which women thought that Malaria (37.5\%), Fever (29.0\%), Cough (27.0\%) and Diarrhea (24.0\%) were also the diseases aimed for prevention in EPI schedule. TB (48.0\%), which is a significantly endangering disease, was reported less than $50 \%$.

Only $212(53.0 \%)$ out of a total of 400 women knew that the vaccination included in EPI program are to be begin from birth of the child. The most common apprehension (10.0\%) against vaccination was that the women thought vaccination might be the cause of illnesses in their children.

From our study, the level of awareness can be distinctively correlated with the level of education of these women. Almost $80.0 \%$ of the women had some fair deal of literacy rate [FIG-2]. This could be correlated with $81.5 \%$ of pregnant women reporting that they are aware of childhood immunization.
A striking improvement in the attitude of Pakistani women was that there was no statistics of gender discrimination regarding vaccination of their children which in past years has been a thought provoking problem.

\begin{tabular}{|l|c|c|c|c|}
\hline \multicolumn{5}{|c|}{ Vaccination for diseases } \\
\hline & Frequency & $\%$ & Frequency & $\%$ \\
\hline Measles & 238 & 59.5 & 162 & 40.5 \\
\hline Malaria & 150 & 37.5 & 250 & 62.5 \\
\hline T.B & 192 & 48.0 & 207 & 51.8 \\
\hline Mumps & 93 & 23.3 & 307 & 76.8 \\
\hline Fever & 116 & 29.0 & 284 & 71.0 \\
\hline Cough & 109 & 27.3 & 291 & 72.8 \\
\hline Diarrhea & 96 & 24.0 & 304 & 76.0 \\
\hline Tetanus & 152 & 38.0 & 248 & 62.0 \\
\hline Hepatitis & 204 & 51.0 & 196 & 49.0 \\
\hline
\end{tabular}

Table-I. Level of knowledge about EPI

\section{Education}

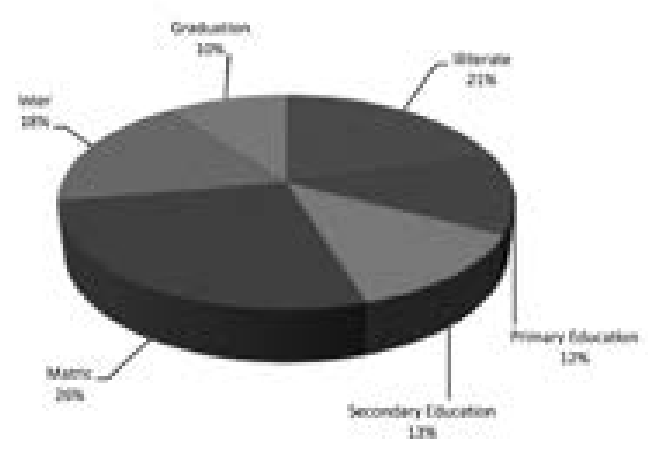

Fig-2. Literacy rate subjects

\section{DISCUSSION}

During the past two-three decades, a considerable amount of statistics and information has become available from a number of developing and already developed countries, showing that maternal education have a strong impact on infant and child mortality. ${ }^{111}$ With effective scientific interventions, a control over such diseases has been put into light with the advent of vaccination and immunization. ${ }^{2}$ Uptake and acceptance of childhood immunization does not only depend 
on the availability of its allied services but also on other key factors including the knowledge and attitude of mothers being an important one..$^{1,5}$

In our study, we have seen that maternal level of education plays a critical part in the knowledge and awareness of childhood immunization programs. A significant proportion of $81.5 \%$ pregnant women included in the study were aware of the existence and remarkable importance of childhood immunization. And among them, 80\% of these women had at least some level of literacy. The education levels of the pregnant women were the most significant predictor of healthy immunization behavior. Being educated did not only make them aware but also helped these women acquire specific knowledge regarding the whole Expanded Program of Immunization schedule in Pakistan.

We also looked for the source of information with respect to childhood immunization among the pregnant women. The over all literacy rate of these expecting mothers was not very high, and a fair deal of them had obtained their education about immunization through oral traditions and stories passed down to them by their Families and their Doctors. Most respondents indicated that they learned about childhood immunization from their respective Doctors, Families, Lady Health Workers, and only a few through specific immunization campaigns and advertisement. A similar study was done on Nepalese mothers by the Stanford University. ${ }^{9}$ The results showed that Doctors and Nurses were the least common source of information regarding immunization.

A thorough questionnaire was so made to also know the extent of knowledge of these pregnant women regarding immunization programs. They were mentioned some diseases and asked to point out those included in the Pakistan's EPI schedule. Polio was the most frequent positive answer (82.2\%), followed by Measles and Hepatitis next. The extensive Polio campaigns and advertisement across the entire country and the efforts of health care providers in making Polio vaccines available even at the remote areas of Pakistan, accounts for this glorious victory. This could probably mean that campaigns and advertisements on vaccination against other important troublesome diseases would also spread awareness about them and this could be the future work in health care provision.

Vaccination starts right from birth ${ }^{2}$ and $53 \%$ of the samples included in our study were aware about this fact. This area of interest could also be worked upon as immunization should start from its right time in order to achieve disease eradication goals. Specific misconceptions and apprehensions against immunization were also looked for. The fear of child falling sick (10\%) was the most common one. Cause of sterility and family opposition did not come out to be significant as it was much hypothesized. A research conducted by the University of Oxford, UK, ${ }^{11}$ showed that barriers identified against vaccination were risk of its adverse effects, distrust of those advocating vaccines (including belief in conspiracy), and concerns that vaccines are painful. The difference is quite evident between the people of the two countries with respect to their own specific apprehensions against vaccination.

Gender discrimination in getting their children vaccinated was not found. This might could be because our sampling was majorly based upon urban population. Similar studies if carried out even in the rural area of Pakistan might be able to bring out alarming results.

Our cross-sectional research survey, brought to light a lot of useful information, in which the sample which was chosen showed quite some positive results. The level of awareness of the respondents with regard to childhood immunization was high, much more than anticipated. Education levels of the pregnant women corresponded to their level of awareness. Further studies could be carried out with a greater sample size and also samples taken from different areas of Pakistan representing more of the general population. This would bring out a more generalized result for the attitude and behavior of Pakistani pregnant women regarding immunization. Level of awareness can also be 
assessed with respect to the trimester of the pregnancy of women, this could give both an idea and comparison as to how much a pregnant woman get aware about immunization with time passing by with her pregnancy.

\section{CONCLUSION}

From our study results, we concluded that maternal education plays a key role towards healthy behavior of childhood immunization among pregnant women. Improvement in the literacy rate as well as massive immunization campaigns might help in the spread of awareness of childhood immunization programs, and its undeniable importance. With achieving this, we would improve upon the preventive measures also along side the curative, in fighting against the battle of disease eradication.

\section{Copyright(C) 03 Dec, 2015.}

\section{REFERENCES}

1. Streatfield K, Singarimbun M, Diamond I., Maternal education and child immunization. Demography. 1990 Aug; 27(3):447-55. http://www.ncbi.nlm.nih.gov/ pubmed/2397822 (Date of access: 12/03/12).

2. Hanan Abbas Abdo Adel Rehman El Gammal. Maternal satisfaction about childhood immunization in primary health care centre, Egypt. Pan Afri Med J 2014;18:157. Published online 2014 June 18.

3. World Health Organization. Estimated Annual Deaths from Diseases that are Preventable by Vaccination. 2010.Available Online:http://www.who.int/immunization/ monitoring__ surveillance/burden/estimates/en/ (accessed on 9 April 2014).

4. Shuaib FM, Kasimbrough D, Roofe M, McGwin G Jr, Jolly P, Factors associated with incomplete childhood immunization among residents in St. Mary, Jamaica. West Indian Med J. 2010 Oct; 59(5):549-54 .http://www. ncbi.nlm.nih.gov/pubmed/21473405 (Date of access: 12/03/12).

5. Daniel A. A.,Olanrewaju O., Folasade E. A., Chris A., Uptake Of Childhood Immunization Among Mothers Of Under-Five In Southwestern Nigeria. The Internet Journal of Epidemiology. 2009; (2). DOI: 10.5580/ f4. http://business.highbeam.com/436084/article1G1-215686634/uptake-childhood-immunizationamong-mothers-underfive. (Date of access: 13/03/12).

6. Velan B. Acceptance on the move: public reaction to shifting vaccination realities, Hum Vaccin. 2011 Dec;7(12):1261-70. http://www.ncbi.nlm.nih.gov/ pubmed/22108039. (Date of access: 12/03/12).

7. Mills E, Jadad AR, Ross C, Wilson K. Systematic review of qualitative studies exploring parental beliefs and attitudes toward childhood vaccination identifies common barriers to vaccination. J Clin Epidemiol. 2005 Nov; 58(11):1081-8. http://www.ncbi.nlm.nih.gov/ pubmed/ 16223649. (Date of access: 12/03/12).

8. Luman E..T; McCauley M. M; Shefer. A;Chu, S.Y. Maternal characteristics associated with vaccination of young children. Pediatrics 2003;111:1215-18.

9. Donald Matsuda, Beliefs About Immunization and Children's Health Among Childbearing Mothers in Nepal. http://www.stanford.edu/group/beagle2/ student_reports/DonnieThesis.pdf (Date of access: $15 / 03 / 1 \overline{2})$.

10. Huntington D, Aplogan A, The integration of family planning and childhood immunization services in Togo. Stud Fam Plann. 1994 May-Jun;25(3):176-83. http://www.jstor.org/stable/2137943. (Date of access: 12/03/12).

11. Polland JA. Childhood immunization: what is future? Arch Dis Child 2007;92:426-33.

\section{AUTHORSHIP AND CONTRIBUTION DECLARATION}

\begin{tabular}{|c|l|}
\hline Sr. \# & \multicolumn{1}{|c|}{ Author-s Full Name } \\
\hline 1 & Dr. Syed Aoun Muhammad Jafr \\
2 & Dr. Iram Saddiqa Aamir \\
3 & Dr. Marium Abid \\
4 & Dr. Maliha Hanif \\
\hline
\end{tabular}

Contribution to the paper

Original research work,

Data collection and Data analysis

Results Interpretation Critical analysis of article especially discussion

Review of results and discussion
Author $=\mathbf{s}$ Signature
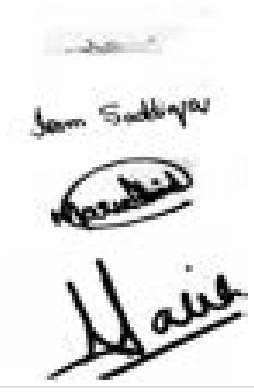